\title{
Real Time Simulation of Security Systems using Face Recongnition, a review
}

\author{
Manisha Sajwan \\ DIT University \\ Dehradun, India
}

\begin{abstract}
In recent years face recognition has received substantial attention from both research communities and the market, but still remained very challenging in real applications. A lot of face recognition algorithms, along with their medications, have been developed during the past decades. In this paper we are going to present reviews about real time simulation of various security systems. These security systems are based on face detection, face tracking and face recognition. In this paper we will also present applications of real-time systems and some advancement that can be done in future to make security systems much faster. There are various algorithms available for face detection, tracking and recognition of human expressions This paper is the review of research work "spontaneous and different facial expressions and poses of individual person faces are detected and stored in the database of organization by giving appropriate and unique card id number and password. If a person comes for work then his or her face is detected and this detected face image is compared with images in the database of that particular organization and unique card id number and password. If the face image and id number are recognized then person is allowed to enter. If it is not recognized then person is not allowed to enter. An alert message is generated to administrator's cell phone and mail to the administrator's mail id. In the reverse we are changing the password by using random string generation and shut down the system".
\end{abstract}

Key-Words: Real-Time Simulation, Survey on Face recognition approaches, Application of Real-Time Security Systems.

\section{1: INTRODUCTION:}

The very first question that comes in our mind is that what is real time simulation? Real-time is a quantitative notion of time. Real-time is measured using a physical (real) clock. Whenever we quantify time using a physical clock, we deal with real time. In contrast to real time, logical time (also known as virtual time) deals with a qualitative notion of time and is expressed using event ordering relations such as before, after, sometimes, eventually, precedes, succeeds, etc. While dealing with logical time, time readings from a physical clock are not necessary for ordering the events.

A system is called a real-time system, when we need quantitative expression of time (i.e. real-time) to describe the behaviour of the system. This paper is basically a review paper or a survey paper for real time simulation of a security system using face recognition techniques for spontaneous and different human face expressions.

This paper is organised as follows:

Section 2 is the history behind face recognition and detection. Section 3 is the brief explanation of face recognition system. Section 4 outlines the real time system basic model. Section 5 describes conclusion and the last section is the outline of references.

\section{2: LITERATURE SURVEY:}

Studies on Facial Expressions and Physiognomy date back to the early Aristotelian era (4th century BC). Physiognomy is the assessment of a person's character or personality from their outer appearance, especially the face. But over the years, while the interest in Physiognomy has been waxing and waning, the study of facial expressions has consistently been an active topic.

Face recognition is one of the applications of image analysis. In 2009, a simple search on "Face Recognition" in the IEEE Digital Library throws 9422 results 1332 articles. Examples are Video surveillance, human-machine interaction, photo cameras, and virtual reality. Face recognition is a relevant term in pattern recognition, neural networks, computer graphics, image processing and psychology.

In the 1950's in psychology the work on this subject hasbeen made. They belong to other issues like face expressions, emotions and perception.

The research on this subject was Woodrow W. Bledsoe. During 1964 and 1965, Bledsoe, along with Helen Chanand Charles Bisson, worked on to recognize faces using computers.

He continued later his researches at Stanford Research Institute. Bledsoe designed and implemented a semi-automatic system. Some face coordinates were selected by a human operator, and then computers used this information for recognition. Face recognition allows variation in illumination, head rotation and aging. Researches trying to measure subjective face features as ear size or between-eye distance. In 1973, Fischler and Elschanger tried to measure similar features automatically.

Algorithm used local template matching to measure facial features. There were approaches back on the 1970's. A face as a set of geometric parameters and based on those parameters 
performs some pattern recognition. In 1973 Kenade was developed a fully automated face recognition system. Kenade compares this automated extraction to a human or manual extraction, showing only a small difference. He got a correct identification rate of $45-75 \%$.

In 1986, the Eigen Faces in image processing technique was made by L. Sirovich and Kirby.This methods were based on the Principal Component Analysis. The goal was to represent an image in a lower dimension without losing information, and then reconstructing it.

In 1990's, the recognition of the mentioned Eigen face approach was the first industrial applications. In1992, Mathew Turk and Alex Pentland of the MIT presented a work which used Eigen faces for recognition. Many approaches which has led to different algorithms like PCA, ICA, LDA and their derivatives. That algorithm was able to locate, track the subject's head.

The foundational studies on facial expressions that have formed the basis of today's research can be traced back to the 17 th century.

A detailed note on the various expressions and movement of head muscles was given in 1649 by John Bulwer in his book "Pathomyotomia".

Another interesting work on facial expressions (and Physiognomy) was by Le Brun, the French academician and painter. In 1667, Le Brun gave a lecture at the Royal Academy of Painting which was later reproduced as a book in 1734. It is interesting to know that the 18 th century actors and artists referred to his book in order to achieve "the perfect imitation of 'genuine' facial expressions". The interested reader can refer to a recent work by J. Montagu on the origin and influence of Le Brun's lectures.

Moving on to the 19th century, one of the important works on facial expression analysis that has a direct relationship to the modern day science of automatic facial expression recognition was the work done by Charles Darwin. In 1872, Darwin wrote a treatise that established the general principles of expression and the means of expressions in both humans and animals.

The first step towards the automatic recognition of facial expressions was taken in 1978 by Suwa et al. By the late 1980s and early 1990s, cheap computing power started becoming available. This led to the development of robust face detection and face tracking algorithms in the early 1990s. At the same time, Human-Computer Interaction and Affective Computing started gaining popularity. Researchers working on these fields realized that without automatic expression and emotion recognition systems 4 , computers will remain cold and unreceptive to the users' emotional state. All of these factors led to a renewed interest in the development of automatic facial expression recognition systems

Since the 1990s, (due to the above mentioned reasons) research on automatic facial expression recognition has become very active. Comprehensive and widely cited surveys by Pantic and Rothkrantz (2000) and Fasel and Luttin (2003) are available that perform an in-depth study of the published work from 1990 to 2001.

Humans communicate effectively and are responsive to each other's emotional states. Computers must also gain this ability. This is precisely what the Human-Computer Interaction research community is focusing on: namely, Affective Computing. Expression recognition plays a significant role in recognizing one's affect and in turn helps in building meaningful and responsive $\mathrm{HCI}$ interfaces. We can refer to Zeng et al.'s comprehensive survey to get a complete picture on the recent advances in Affect-Recognition and its applications to HCI.

Practical real-time applications have also been demonstrated. Bartlett et al. have successfully used their face expression recognition system to develop an animated character that mirrors the expressions of the user (called the $C U$ Animate)

The various facial behaviours and motions can be parameterized based on muscle actions. This set of parameters can then be used to represent the various facial expressions. Till date, there have been two important and successful attempts in the creation of these parameter sets:

\section{3: A GENERIC FACE RECOGNITION SYSTEM:}

The input of a face recognition system is always an image or video stream. The output is an identification or verification of the subject or subjects that appear in the image or video. Some approaches define a face recognition system as a three step process. From this point of view, the Face Detection and Feature Extraction phases could run simultaneously. Face detection is defined as the process of extracting faces from scenes. So, the system positively identifies a certain region as a face. The procedure has many applications like face tracking, pose estimation or compression.

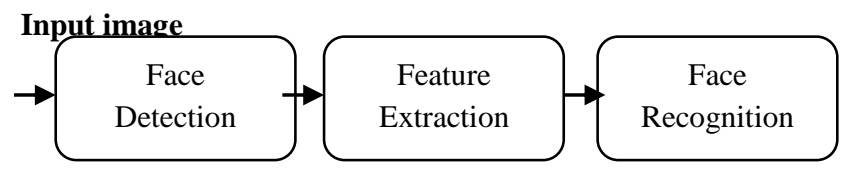

Fig: Face recognition System

The next step -feature extraction- involves obtaining relevant facial features from the data. These features could be certain face regions, variations, angles or measures, which can be human relevant (e.g. eyes spacing) or not. This phase has other applications like facial feature tracking or emotion recognition.

Finally, the system does recognize the face. In an identification task, the system would report an identity from a database. This phase involves a comparison method, a classification algorithm and an accuracy measure. This phase uses methods common to many other areas which also do some classification process -sound engineering, data mining. 
These phases can be merged, or new ones could be added. Therefore, we could find many different engineering approaches to a face recognition problem. Face detection and recognition could be performed in tandem, or proceed to an expression analysis before normalizing the face.

\section{4: A BASIC REAL-TIME SYSTEM MODEL:}

We need to have a basic conceptual understanding of the underlying hardware. We therefore in this section try to develop a broad understanding of high level issues of the underlying hardware in a real-time system.

Following Figure shows a simple model of a real-time system in terms of its important functional blocks. Unless otherwise mentioned, all our subsequent discussions would implicitly assume such a model.

Observe that in Figure the sensors are interfaced with the input conditioning block, which in turn is connected to the input interface. The output interface, output conditioning, and the actuator are interfaced in a complementary manner.

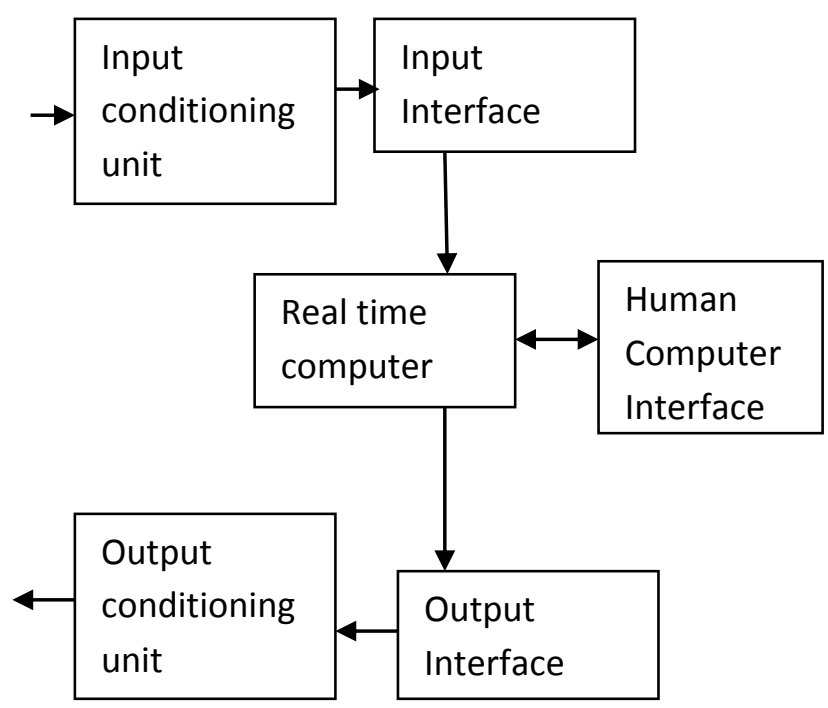

Fig: Basic Model of Real-time system

\section{5: CONCLUSION:}

This paper's objective was to introduce the real time security system using face expression recognition and the associated areas in a manner that should be understandable even by the new comers who are interested in this field but have no background knowledge on the same. In order to do so, we have looked at the various aspects of face expression recognition in detail. We have many applications that have been implemented and other possible areas where automatic expression recognition can be applied.
Face expression recognition systems have improved a lot over the past decade. The focus has definitely shifted from posed expression recognition to spontaneous expression recognition. The next decade will be interesting since I think that robust spontaneous expression recognizers will be developed and deployed in real-time systems and used in building emotion sensitive HCI interfaces. This is going to have an impact on our day to day life by enhancing the way we interact with computers or in general, our surrounding living and work spaces.

Artificial neural networks are a popular tool in face recognition. They have been used in pattern recognition and classification. Kohonen was the first to demonstrate that a neuron network could be used to recognize aligned and normalized faces.

Testing of algorithm will be using different additional bias constraints to obtain better results. By decreasing the error rate we can achieve better outputs, although it is more time-consuming that the simple method.

In future Speech recognition and speech processing can be embedded with image processing to make our security system efficient and effective Detecting the motion of the speech is not that easy as it seems to be. Besides human facial expressions speech has proven as one of the most promising modalities for the automatic recognition of human, but accuracy is the major factor of the speech processing. I would involve the classification of voice file using new classification method with combinational of algorithms such as MFCC, SVM and Neural network.

\section{6: REFERENCES:}

[1] A Survey of Face Recognition Techniques,Journal of Information Processing Systems, Vol.5, No.2, June 2009

[2] Introduction to real time systems, version 2, IIT kharagpur.

[3] Face Recognition Algorithms,Proyecto Fin de Carrera, June 16, 2010 ,Ion Marques,Supervisor: Manuel Grana.

[4] Hadid, "The local binary pattern and its applications to face analysis," in Proc. Int.Workshops Image Process. Theor., Tools Appl., 2008, pp. 28-36.

[5] Bhumika G. Bhatt , Zankhana H. Shah Face Feature Extraction Techniques: A Survey National Conference on Recent Trends in Engineering \& Technology 2013

[6] B. Froba and A. Ernst, "Face detection with the modified census transform," in Proc. IEEE Int. Conf. Autom. Face Gesture Recog., 2004, pp. 91-96.

[7] Chennamma, H. R., Ragrajan, L., Rao, M. S. Robustnearduplicate image matching for digital image forensics. International Journal of Digital Crime and Forensics, 2009 vol. 1 , no. 3,18 p.

[8] Mohamed A. Berbar ,Hamdy M. Kelash, and Amany A. Kandeel, Faces and Facial Features Detection in Color Images, Proceedings of the Geometric 
International Journal of Computer Applications Technology and Research

Volume 4- Issue 2, 129 - 132, 2015, ISSN:- 2319-8656

[9] Modeling and Imaging - New Trends (GMAI'06) 0-76952604-7/06 \$20.00 @ 2006 IEEE

[10] Mrs.Smita Patil Prof. Mrs. A. A. Junnarkar, Overview of Colour Image Segmentation Techniques, IJARCSSE Volume 3, Issue 9, September 2013, ISSN: 2277 128X

[11] Nikita Sharma, D.S.Singh, A Survey on various Feature Extraction Techniques for Face Recognition, IJCA, ISSUE 2, Volume 4 (August 2012), ISSN: 2250-1797, Page 18

[12] Roopashree.S, Sachin Saini, Rohan Ranjan Singh. Enhancement and Preprocessing of Images using filtering, IJEAT, ISSN: 2249-8958 Volume-1 Issue-5,

[13] TusharGajame, C.L. Chandrakar Face Detection with Skin Color Segmentation \& Recognition using Genetic Algorithm International Journal of Innovative Technology and Exploring Engineering (IJITEE) ISSN: 2278-3075, Volume-3, Issue-2, July 2013

[14] K. Sung and T. Poggio, Example-based Learning for View-based Human Face Detection, A.I. Memo 1521, MIT A.I. Laboratory, 1994.

[15] Miss.Renke Pradnya Su nil Automatic Face Recognition Using Principle Component Analysis with DCT, Journal of Electronicsl and Communication Engineering (IOSR-JECE2013) ISSN: 2278- 2834- , ISBN: 2278-8735, PP: 01-07. 